\title{
Morphological and behavioural variation in CNS innate defence cell microglia is development and age sensitive
}

\author{
Payel Ghosh, Anirban Ghosh
}

Department of Zoology and Immunobiology Laboratory, Panihati Mahavidyalaya, West Bengal State University, Sodepur, Kolkata 700110, West Bengal, India.

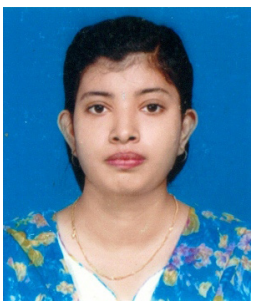

Payal Ghosh is a Junior Research Fellow holding the prestigious UGC-NET fellowship from MHRD, Government of India and working with Dr. Anirban Ghosh for over three 3 years. She is involved to find out the colonization and morphofunctional pattern of microglia with its cellular adaptability throughout the developmental continuum in rodent model.

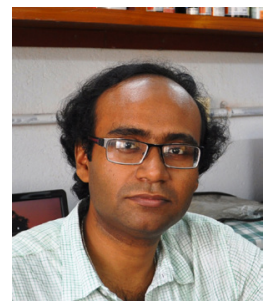

Dr. Anirban Ghosh is working on neuroimmunology for last 15 years. Also, he is interested on microglia and its morpho-functional features, colonization pattern etc. in brain development and maturity, as well as the contribution of microglia in glioma restriction and/ or progression. His research is supported by different Government of India funds like UGC, SERB, ICMR \& CSIR and he has considerable publications throughout the period in the field.

\section{A B S T R A C T}

Aim: Microglia, the innate defence cells in central nervous system (CNS), alters their shapes and function with age. We observed and identified these morphological changes and functional association throughout the developmental gradient until adulthood in rat brain. Methods: Early and late embryonic stages, neonates and adult brains of albino rats were sectioned for routine Haematoxylin Eosin (HE) staining and specialized silver-gold staining to show distribution and morphological variation in situ. Isolated microglia from different age groups was subjected to scanning electron microscopy (SEM) for observing ultrastructural shapes of microglial cells. The Viability of isolated cells was measured by trypan blue staining and their cellular identity by immuno-staining for CD11b. Finally, phagocytic limitations of the cells in normally developing brain were assessed by carbon particle ingestion and oxidative burst through nitroblue tetrazolium assay to investigate microglial age-sensitivity behavioural response. Results: HE staining spotted overall cellular distribution in the brain and cells with monocytic appearance among the other CNS cells. On contrary, silver-gold staining showed variable morphologies of microglia in various age groups and also showed the appearance of ramified microglia in adult. Nearly $90 \%$ of isolated cells were viable and positive for CD11b. SEM showed variable shapes of amoeboid and ramified forms. Immunofluoresence confirms microglial identity. Functionally, microglia showed an age dependent baseline phagocytic capacity in normal condition which changes with developmental phase and age with most active phagocytic behaviour around perinatal phase. Conclusion: In normally developing brains, microglia shows variability in morphology and baseline phagocytic activity that changes with age. These results may represent the normal physiology of CNS development and function.

Key words: Microglia; central nervous system; development; phagocytosis; nitroblue tetrazolium assay

\section{INTRODUCTION}

Among the ectodermal allies in brain there are some mesodermal aliens spread throughout the tissue. They

Corresponding Author: Dr. Anirban Ghosh, Department of Zoology and Immunobiology Laboratory, Panihati Mahavidyalaya, West Bengal State University, Barasat Road, Sodepur, Kolkata 700110, West Bengal, India. Email: aghosh06@gmail.com.

\begin{tabular}{|l|l|}
\hline \multicolumn{2}{|c|}{ Access this article online } \\
\hline Quick Response Code: & Website: \\
\hline & www.nnjournal.net \\
\hline & \\
& \\
\hline
\end{tabular}

are myeloid/monocytic lineage cells and acting as the sentinel of this delicate organ on behalf of the innate immunity of the body. Although there is a consensus about the myeloid origin of microglia, much controversy remains regarding the precise nature of microglial progenitors. Many authors claim that microglial cells arise early during development from progenitors in

This is an open access article distributed under the terms of the Creative Commons Attribution-NonCommercial-ShareAlike 3.0 License, which allows others to remix, tweak, and build upon the worknon-commercially, as long as the author is credited and the new creations are licensed under the identical terms.

For reprints contact: service@oaepublish.com

Cite this article as: Ghosh P, Ghosh A. Morphological and behavioural variation in CNS innate defence cell microglia is development and age sensitive. Neuroimmunol Neuroinflammation 2016;3:38-47.

Received: 25-07-2015; Accepted: 04-11-2015 
the embryonic yolk sac that are then seeded in the rudimentary brain and persist there into adulthood. ${ }^{[1]}$ Another school of scientists thought that their entry in brain from blood also occurs at some points in embryonic and early natal phases, which also gradually populates into entire brain and designated as microglia. ${ }^{[2-5]}$ Microglia function like a hybrids of glia and leukocytes and thus express a variety of cytokine receptors as well as producing cytokines themselves. ${ }^{[6]}$ Microglia forms an extremely stable population in brain and comprise up to $20 \%$ of total glial population forming an immune accessory network..$^{[7,8]}$ Microglia can adapt according to the central nervous system (CNS) microenvironment, monitor the CNS integrity and also act under the strict control of neurobiochemical environment. Recent studies indicate that they function in maintaining normal tissue homeostasis in brain at the resting state through scanning their territorial domains. ${ }^{[9-11]}$ They phagocytose cellular debris, contribute to restructuring neuronal circuits and triggering repair, which are assumed to be related with development. ${ }^{[12-16]}$ Depending on specific environmental context microglia play a dual role of neuroprotection or neurotoxic..$^{[12,17-19]}$

There are few studies which showed that microglial morphology changes with age, but most of the studies have dealt with their changes in neuropathological conditions. ${ }^{[20]}$ Microglial transformation from ramified to amoeboid affects their functional modifications. The effector role of the cells are mostly studied in disease models or subjects, ${ }^{[21]}$ But seldom has any attempt been made to evaluate the baseline physiological response of the cells in a normally developing brain beginning at birth. However, recent findings showing their role

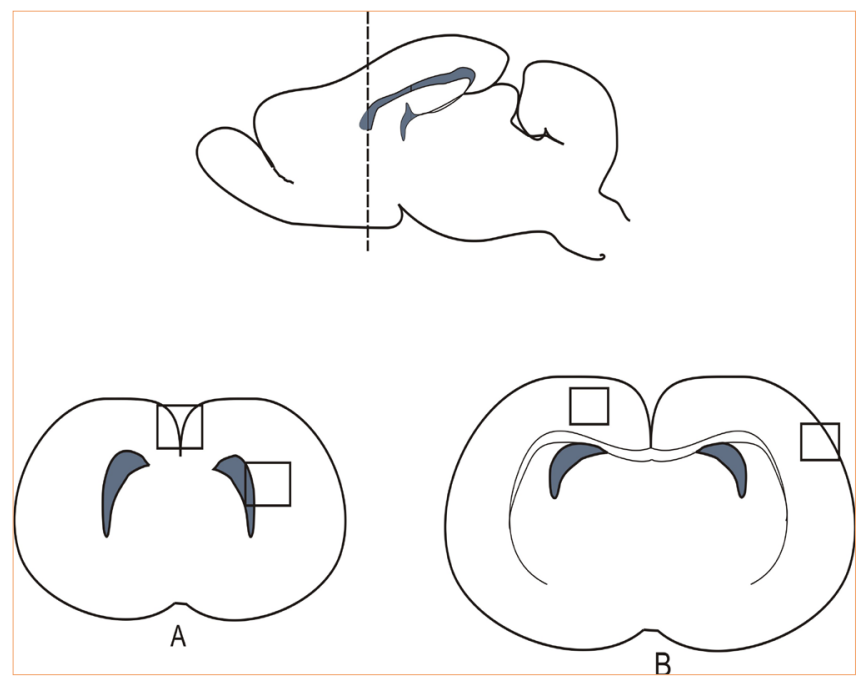

Figure 1: Graphical representation of a rat brain showing the sagittal and coronal view of the section planes. Dotted line in the sagittal view shows the plane of section used for histology and filled grey areas are showing lateral and 3rd ventricles. Diagram marked A shows the coronal view of late embryonic and neonatal pups where square boxes are showing the sectioned areas adjacent to lateral ventricles and neocortex at the cleavage of two hemispheres which are the areas represented in the photomicrographs. Diagram B shows the corona view of adult rats where square boxes are showing the regions of cerebral cortex represented here in neuronal circuit development and maintaining tissue homeostasis indicates that they have a basal physiological function from developing embryo to growing adult. ${ }^{[11,13]}$ In the present study our attempt is to find that basal morphological and behavioural variation of microglia from embryo to growing brain up to its maturation, excluding aging brain. With identifying in situ distribution and morphological transformation, we also isolated them to assess the morphological differences and functional deviation in terms of phagocytosis.

\section{METHODS}

\section{Animal and grouping}

The Sprague-Dawley rats were maintained for the experiment as approved by institutional animal ethical committee (Approval No. -AG/CP/IAECWBSU/2011-12/5) and according to the animal experiment procedures strictly followed the "Principles of Laboratory Animal Care” (NIH publication no. 8523, revised in 1985). The animals were fed with pellet diet, or equivalent, and water ad libitum, $12 \mathrm{~h}$ light and dark cycle were maintained, examined and weighed at regular interval throughout the experimental period. Reproductively matured male and receptive female were set for breeding at a rate of 1:2 respectively, examined for confirmation of mating usually made by visualising the copulatory plug, after that pregnant mothers were separated and pregnancy days were counted to obtain the required embryos. ${ }^{[22]}$ Neonates were maintained with their respective mothers in one cage as they were at waning age. The groups of animals maintained were (1) early embryo (ED $10 \pm 1$ ); (2) late embryo (ED $18 \pm 2$ ); (3) neonate (D $5 \pm 1$ ); (4) young adult (D45 \pm 5) and (5) mature adult (D $240 \pm 10$ ).

Histological sectioning of brain tissue and haematoxylineosin staining

The rats were deeply anaesthetized with sodium pentobarbital (50 mg/kg body weight). The whole brain was dissected out, initially placed in ice cold Phosphate Buffer Saline (PBS) and then postfixed in 10\% buffered formalin (NICE, India) for overnight at $4{ }^{\circ} \mathrm{C}$. After fixation, foetal, postnatal and adult brains were washed in PBS, dehydrated through graded alcohol (30\%, 50\%, 70\%, 95\% and absolute alcohol) and embedded in paraffin (MERCK, India) through histokinate processing. From this block coronal sections of brain were cut at 5-7 $\mu \mathrm{m}$ thickness with a microtome (WESWOXTM OPTIK Rotary Microtome, Model-MT-1090A, India). The sagittal and coronal sections [Figure 1] show the schematic positions of the rat brain cerebral cortex adjacent to the ventricular margin and inner and outer cortex which are used and represented in the study. The sections were then routinely stained with haematoxylin-eosin (HE) 

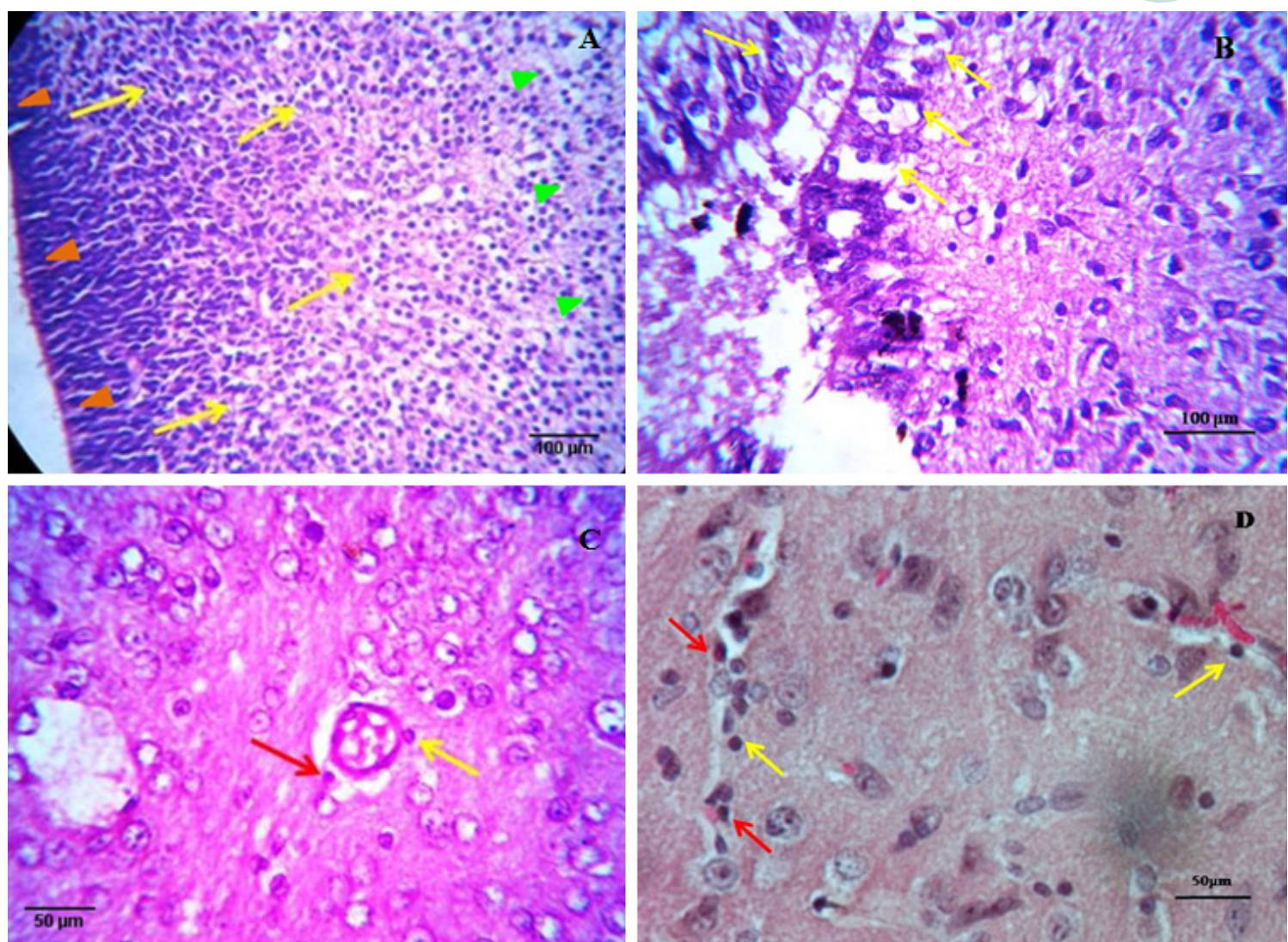

Figure 2: Haematoxylin-eosin stained brain sections of early embryo $(\times 40)(A)$, late embryo $(\times 40)(B)$, neonate $(\times 40)(C)$ and young adult $(\times 100)(D)$. $(A)$ Different neuroglia precursor cells and a huge number of predicted myeloid cells of forming ventricular margin radiate towards outer cortex and colonize there. Migration was indicated by arrows (yellow), ventricular margins by orange arrowheads and outer cortex by green arrowheads; (B) a distinct band of cells with a definite organisation appears at the margin (indicated by arrows) but immediately after that diffused cell matrix with scattered cells is observed; (C) in case of neonates along with oligodendrocytes, astrocytes, neuronal cells, a prominent blood capillary with extravasation (indicated by red arrow) of amoeboid monocytic cells out of the BBB is visible. A cell also tethered to the margin of the capillary (indicated by yellow arrow); (D) gathering (indicated by red arrows) and infiltration (indicated by yellow arrows) of blood vessel containing leukocytes into the brain parenchyma to form a stable population of monocytic cells. Perivascular macrophage/microglia are also observed

to identify and observe the distribution of overall cell population in brain. Selected fields were documented by Olympus DSC (12 Megapixel) camera through Olympus CH20i Microscope and processed by Image J software (NIH, USA).

\section{Silver gold staining of brain tissue}

Histological sectioning of brain tissues [Figure 1] were then subjected to a specialized silver carbonate staining first introduced by del Rio Hortega (1918) and gold toning by Penfield (1937), modified by McCarter (1939). ${ }^{[23,24]}$ Briefly, $10 \mu \mathrm{m}$ thick brain sections were initially deparaffinised by using xylene (MERCK, India) and placed in aqueous ammonia solution, then passed through Globus' hydrobromic acid (MERCK, India) solution and washed. The slides were then rinsed into $50 \%$ aqueous solution of silver carbonate derived from silver nitrate (FINAR, India) and sodium carbonate (CDH, New Delhi) for $1 \mathrm{~h}$. Sections were then passed in formalin and washed and placed in aqueous gold chloride (HIMEDIA, India) to counter-stain. After that, slides were rinsed thoroughly in $\mathrm{dH}_{2} \mathrm{O}$ and fixed in sodium thiosulfate (MERCK, India) solution, washed, dehydrated in alcohol and mounted in DPX
(MERCK, India) to observe under the microscope. The morphological changes of microglia in situ from embryonic to adult normal brains were documented with the microscope Nikon Eclipse TS 100, using CCD Camera (DS-Fi2-U3) and analysed by NIS Elements BR software (Nikon Corporation, Japan).

Isolation of microglia from different age groups of brains

Microglia were isolated and characterized as described previously with slight modification. ${ }^{[25]}$ Briefly, after heart perfusion of anesthetized rats, dissected brain tissues wereplaced underabinocularstereomicroscope(Magnus MS-24) to peel off major blood vessels and capillaries. The whole brain was mechanically dissociated, lightly homogenized and enzymatically digested for $30 \mathrm{~min}$ at $37^{\circ} \mathrm{C}$ by $5-15 \mathrm{U}$ type II collagenase (Sigma-Aldrich, USA) and 500 U DNase I (Sigma-Aldrich, USA). ${ }^{[26]}$ Then the suspension are washed and resuspended in ice cold PBS and passed through stainless steel sieve of porosity about $80 \mu \mathrm{m}$ to make single cell suspension. The cell suspensions were then allowed to adhere on glass petri-dish (DURAN, Czech Republic) for $1 \mathrm{~h}$ in $5 \% \mathrm{CO}_{2}$ humified environment at $37^{\circ} \mathrm{C}\left(\mathrm{CO}_{2}\right.$ Incubator Galaxy 48S, New Brunswick, Germany). The adherent cells were then recovered with Trypsin-EDTA (MP Biomedicals, 

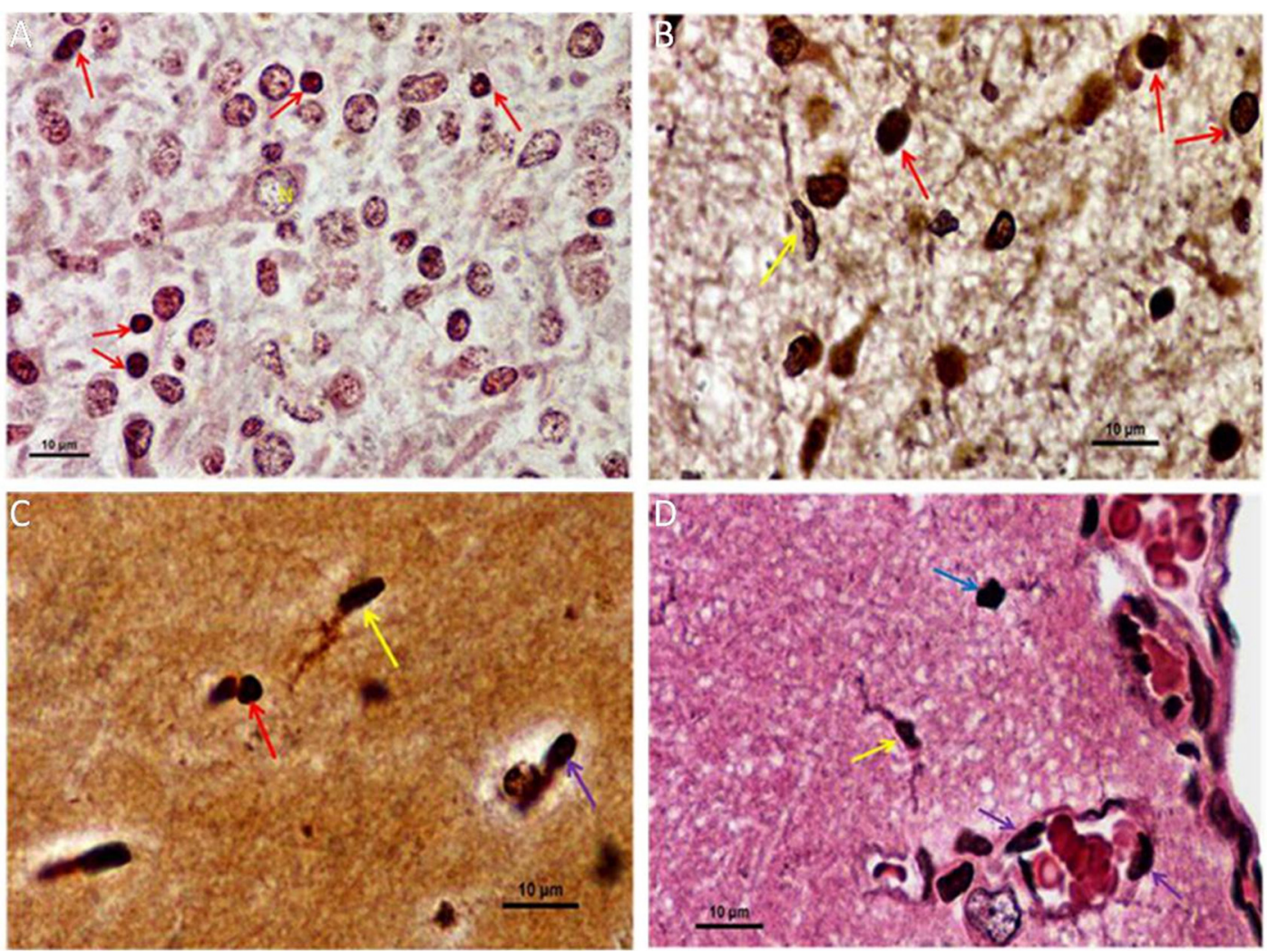

Figure 3: Silver gold staining of brain tissue showing morphological variation of microglia/macrophage in both developing and adult rat brain ( $\times 100$, Oil immersion). (A) In late embryo, densely stained myelo-monocytic lineage cells (indicated by red arrows) along with other neuroglial cells and a distinct neuronal cell body are observed in brain tissue matrix. "N" represents nucleus of the neuron; (B) in neonates, mostly amoeboid microglial cells (indicated by red arrows) are evenly distributed throughout the matrix and a distinct ramified microglia (indicated by yellow arrow) is also appeared; (C) whereas in young adult, ramified microglia with their slender ramifications (indicated by yellow arrow) are clearly visible and cells with amoeboid morphology (indicated by red arrow) are also observed. In addition, the entry of a deeply stained cell into the brain matrix from a capillary (indicated by violet arrow) is visible; (D) in late adult, both irregular shaped amoeboid microglia (indicated by blue arrow) and ramified microglia with their projections (indicated by yellow arrow) are clearly found in cortical regions. From blood capillary or vasculature the entry of myeloid cells in brain parenchyma is visible by the presence of deeply stained cells at the margin of capillary (indicated by violet arrow), among them a few cells just entering into the tissue matrix and resides there

USA) solution followed by addition of 1X RPMI-1640 media with $10 \%$ fetal bovine serum (FBS) is used to neutralize the reaction of trypsin-EDTA solution ${ }^{[27]}$ and there after centrifuged, supernatant was discarded and pellet was washed. The resulting cell suspensions were then laid on $20-70 \%$ Percoll gradient at 2,000 rpm for 25 min yielding highly enriched microglia at the interface. Cells were recovered, washed and suspended in RPMI1640 (MP Biomedicals, USA) containing 1\% penicillinstreptomycin (P/S, MP Biomedicals, USA) and their viability was immediately measured by trypan blue exclusion $(10 \mu \mathrm{L}$ trypan with $10 \mu \mathrm{L}$ cell suspended in media) in Neubauer Improved Chamber (Marienfeld, Germany). In cases of embryo and neonates, the whole brain of 5-6 pups or embryos were pooled together and treated with half-dilution of enzyme concentration as for adult rat and some modification in centrifugation. The morphological variation among different groups viability of isolated cells were documented and photographed by Nikon Microscope Nikon Eclipse TS 100, using CCD Camera (DS-Fi2-U3) and NIS Elements BR software (Nikon Corporation, Japan).

Characterisation of microglial cells by immunofluoresence with CD11b marker

Isolated cells were cultured in RPMI 1640 media (contains 10\% FBS + 1\% P/S) for 3 days in a humified $\mathrm{CO} 2$ incubator at $5 \% \mathrm{CO}_{2}$ level at $37{ }^{\circ} \mathrm{C}$. After a $\mathrm{PBS}$ wash cells were mildly fixed with $2 \%$ paraformaldehyde solution for $10 \mathrm{~min}$ at $4{ }^{\circ} \mathrm{C}$ temperature, washed and stained with FITC conjugated fluorescein anti-CD11b antibody (eBioscience, USA) diluted in 1\% FBS $\left(\mathrm{Gibco}^{\circledR}\right.$, Life technologies, USA) in PBS (1:500) and incubated for $1 \mathrm{~h}$ in a dark humified chamber. After washing, the cells were observed under inverted fluorescence microscope (Nikon Eclipse TS 100), photographed using a CCD Camera (DS-Fi2-U3), and analysed by NIS Elements BR software (Nikon Corporation, Japan).

\section{Ultrastructure of microglia in scanning electron microscopy}

The cells immediately isolated from brain tissue were fixed in $2.5 \%$ glutaraldehyde for $4 \mathrm{~h}$ at $4{ }^{\circ} \mathrm{C}$ followed by washing in PBS and gradually dehydrated in graded alcohol and finally brought to $100 \%$ acetone. Dehydration were followed by critical-point drying, spread on $1 \mathrm{~cm}^{2}$ grease-free glass slide placed on metallic stub with conducting silver paint. The cells were laid on a glass platform and then coated with gold-palladium alloy of $100 \AA-200 \AA$ thickness in a diode sputtering system. Then the samples were observed under Scanning Electron Microscope (HITACHI, S530, Japan) at $15 \mathrm{KV}$ beam 
Table I: Age dependent cellular viability (in percentage) as obtained in culture

\section{Groups}

Early embryo (ED $10 \pm \mathrm{I})$

Late embryo (ED $18 \pm 2$ )

Neonates (D $5 \pm 1$ )

Young adult (D $45 \pm 5)$

\section{$\%$ of viable cells \\ $87 \pm 3$ \\ $86 \pm 2$ \\ $90 \pm 3$ \\ $92 \pm 3$}

voltage. The specimens were scanned and photographed.

Phagocytosis and measurement of oxidative burst of microglia by nitroblue tetrazolium chloride reduction assay

Phagocytic properties of the isolated cells from different age groups were observed and quantified parallel by incubating cells with ultrafine carbon particles (SRL, India) and nitroblue tetrazolium chloride (NBT) salt (Sigma, USA). NBT reduction assays with carbon particle ingestion, were performed to visualize phagocytosis and measure the oxidative burst of phagocytic cells. For both early and late embryos, a pool of cells was made from a single mother's foetus or pups. In case of early embryos from a single mother, an average of 7 embryos were obtained from which cells were isolated. The same process was done for another 2 pregnant mothers ( $n=3$ pregnant mother foetus). For late embryos, an average of 6 embryos were isolated from a single mother for an experiment, which was conducted in triplicate ( $n=3$ pregnant mother foetus). In the case of neonates, 3 sets, each containing an average of 6 pups, were taken to execute the experiment $(n=3)$. Whereas in case of young adults as the volume of brain and number of cells obtained from each adult rat was sufficiently higher 3 individuals $(n=3)$ were used for three sets of experiments. In each age group, cell numbers were diluted to $10^{7}$ cells $/ \mathrm{mL}$. The isolated cells along with $0.1 \%$ NBT and ultrafine carbon particle were allowed to incubate at $37{ }^{\circ} \mathrm{C}$ overnight. The reaction was stopped with $0.1 \mathrm{~N}$ chilled $\mathrm{HCl}$. A sample of cells from each reaction mixture was transferred to a Neubauer's chamber to observe under a phase contrast microscope (Nikon Eclipse TS 100) and documented using a CCD Camera (DS-Fi2-U3) and analysed with NIS Elements BR software (Nikon Corporation, Japan). The remainder of each reaction mixture was centrifuged, supernatant removed, pellets resuspended in pyridine, and boiled for $10 \mathrm{~min}$, which converted the extract into blue by reducing NBT into formazan, which can be measured colorimetrically at $540 \mathrm{~nm}$. The OD value is directly proportional to the level of phagocytosis or, more precisely, the reactive oxygen species (ROS) generation, ${ }^{[21]}$ by different developmental age groups.

\section{Statistical analysis}

Microsoft Excel (Redmond, WA) was used to compute and tabulate experimental results. For statistical interpretation, one-way ANOVAs, followed by TukeyKramer post-hoc tests, were performed, and the results were analysed using OriginPro 8 Software and graphically represented. Data were expressed as mean \pm SD and a $P$ value $<0.05$ was considered significant
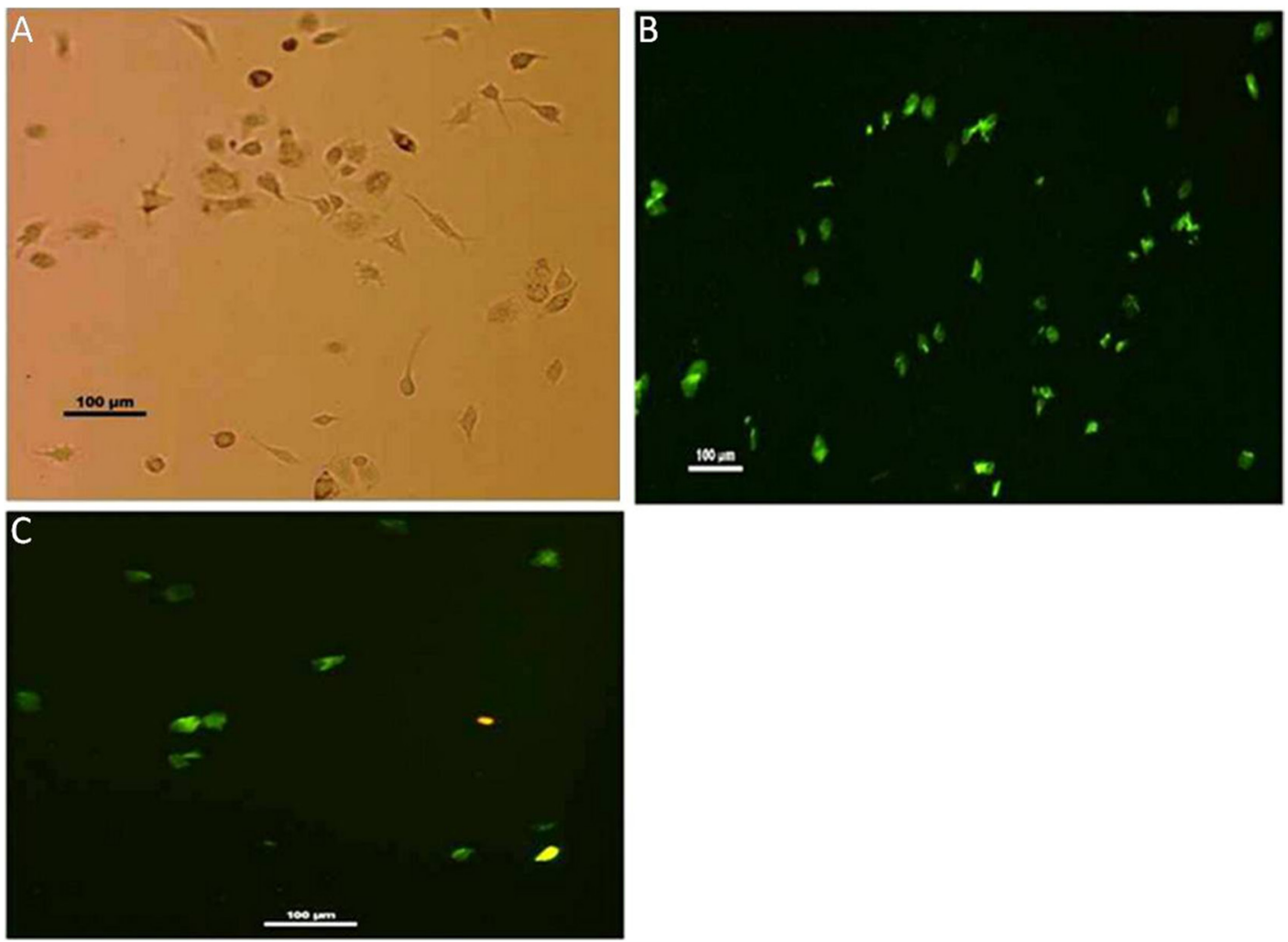

Figure 4: Isolated microglial cells. (A) Microglia isolated from rats shows varied morphology i.e. both irregular and elongated cells with extended pseudopod. ( $\times 10) ;(B)$ immunostaining of isolated cells from adult rat brain showing CD11b+ (positivity) prominently with morphological variations, i.e., both irregular and elongated cells are observed ( $\times 10)$; (C) whereas in case of neonates most of CD11b+ cells are showing irregular morphology $(\times 10)$ 

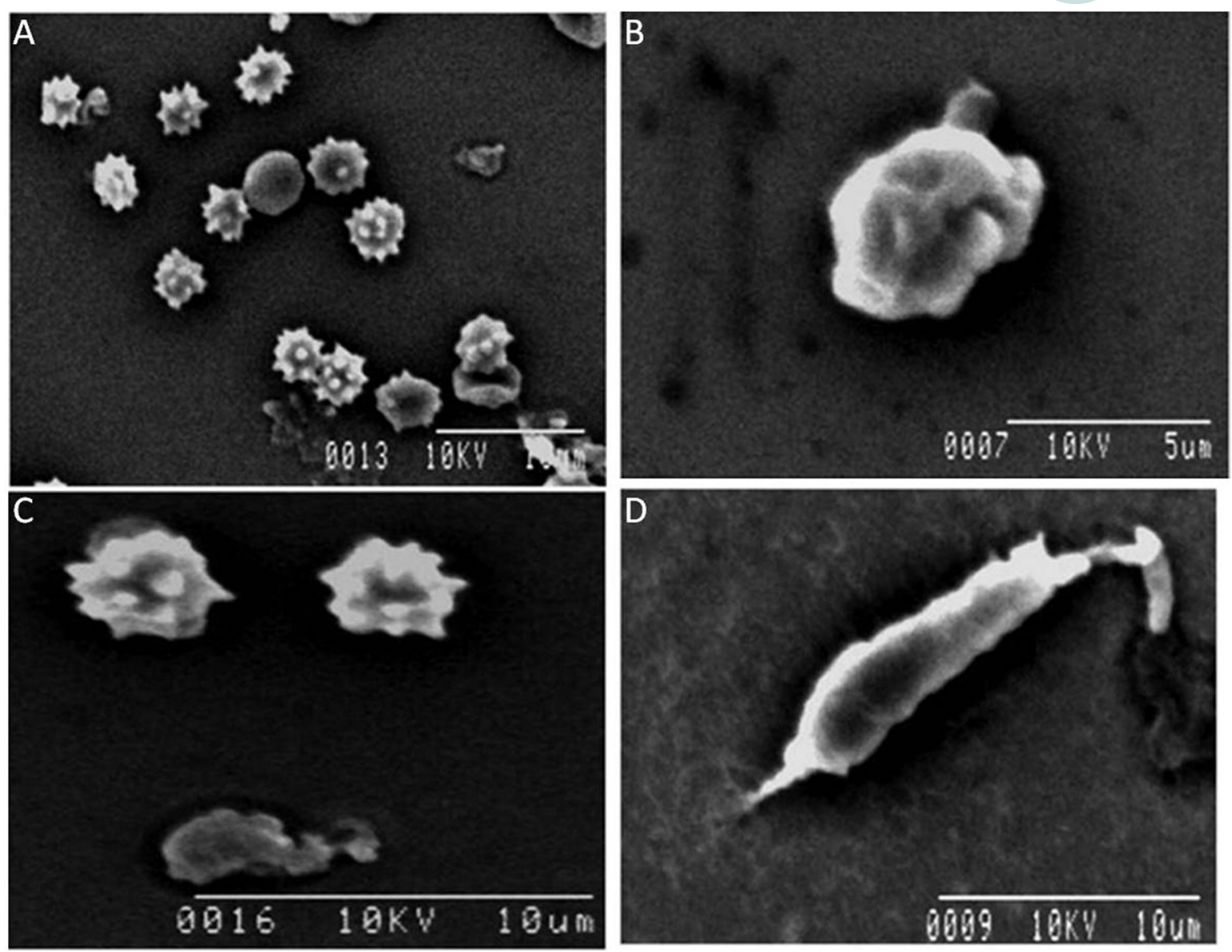

Figure 5: Scanning electron micrograph of isolated microglia cells from brain tissue. (A) Microglia recovered from early embryonic developing brain show many of them retain spines bearing surfaces which radiate out of the cell body; (B) a few cells remain amoeboid in shape with a ruffled surface membrane and short stubby projections in late embryo; (C) in neonates, the presence of both amoeboid cells with spines bearing surfaces and also extended structure of amoeboid microglia cell; (D) ramified or "resting" microglia isolated from adult brain with extended pseudopodia are seen

\section{RESULTS}

Colonization pattern of neuroglial cells from embryo to adult

Staining of brain tissue of different ages with normal HE staining shows a changing cellular distribution in the brain across time. In the early embryo, huge numbers of presumptive neuroglial and myeloid cells were found to migrate from the inner ventricular margin to the outer cortex region. Different glial progenitor cells, along with neuronal precursor cells, colonize the brain parenchyma from the predictive neuroglial stem cell line at the margin of the forming ventricle [Figure 2A]. In the case of a late embryo, normal HE staining shows a remarkably different cellularity near the ventricle. A cellular band is distinct at the margin, but immediately after that, a diffused cell matrix starts with scattered cells. Variations among the cells are more prominent, indicating further differentiation occurring in the cells [Figure 2B]. An example of a section from a neonate of 4-5 days clearly shows perivascular monocyte cells and a cell extravasating out of a blood capillary, hence, across the blood brain barrier (BBB), indicating blood capillaries as important sources of myeloid/ monocytic cells that colonize the brain [Figure 2C]. In a section from an adult, normal HE staining reveals brain parenchyma with a blood vessel containing leukocytes, many of which are present at the margin of the capillary and tethered to the endothelium and therefore extravaseting at the perivascular spaces, along with other neuroglial cells in the matrix [Figure 2D]. Therefore, normal HE staining shows overall cellular distribution, colonization, and differentiation patterns of various brain cells that change remarkably from embryo to adult.

Variable morphological forms of microglia in developing through adult brain

Silver-gold staining of brain tissue, in situ, can differentiate myeloid lineage cells, including microglia/ macrophages, from neuronal and glial populations by its characteristic dark staining relative to background. ${ }^{[28,29]}$ As a result, changes in shape from "amoeboid" in the embryo to "ramified" in the adult, is documented. In early embryos, the developing brain is saturated with presumptive cells that stain darkly and are hard to distinguish between neuro-glial and microglial precursors, and hence, were omitted. In late embryos [Figure 3A], the deeply stained myelo-monocytic lineage cells, or presumptive microglia, appear prominently in comparison with other neuroglial cells. A distinct and prominent neuronal cell body, along with a dendritic 

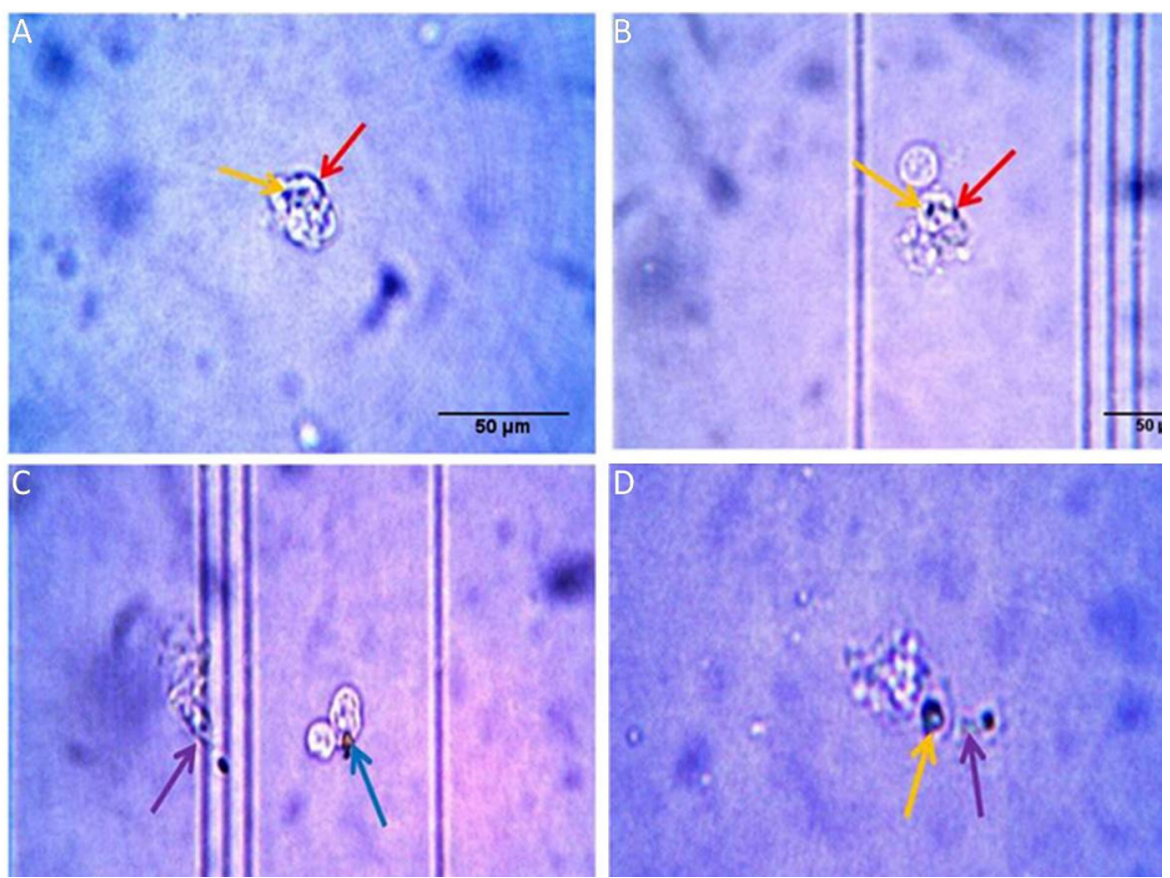

Figure 6: Microglial ingestion of carbon particle in (A) early embryo, (B) late embryo, (C) neonates and (D) adult. In case of both embryonic stages a few carbon particles were attached to the cell (indicated by red arrows) whereas some particles become engulfed by the cell (indicated by yellow arrows). In neonates, a phagocytic vacuole (indicated by blue arrow) with carbon particle is observed along with a cell which engulfs a carbon particle by extending its pseudopodia (indicated by violet arrow). In an adult, an irregular shaped cell shows the same pattern of phagocytotic movement towards a carbon particle; the cell engulfs one particle and also extends a projection towards another carbon particle (indicated by yellow \& violet arrows respectively)

projection, is also observed. In neonates [Figure 3B], microglia cells are found evenly dispersed throughout the brain matrix, along with other neuro-glial cells. Both amoeboid and distinctly ramified microglia, with their long tactile projections, has now appeared. Furthermore, amoeboid microglia predominates in neonatal brain. In the case of 3 months old rat brain or young adult [Figure $3 \mathrm{C}$ ], the so called ramified microglia with their slender projections, elongated cell body, and nucleus are clearly visible and appear high in number. Similarly, cells of amoeboid morphology are also observed. In the mature adult [Figure 3D], microglia, with their ramifications, are found distinctively throughout brain, along with other amoeboid forms. Here, ramified microglia are prominent in the cortical region along with an irregular shaped amoeboid form and the morphological differences are distinctly prominent. At the same time, it is seen that some deeply stained cells tethering with the margin of blood capillary or vasculature, while others just seem to enter in to the tissue matrix. In brief silvergold staining shows that during pre and postnatal phase, amoeboid microglia are present predominantly, and a few ramified form of microglia are detected in postnatal phase. Whereas, in adult, both forms of microglia were observed and a blood capillary distinctly enabling the entry of myeloid cells into brain parenchyma.

Identification, characterisation and measurement of cell viability of isolated microglia

Microglia isolated from rat brain are identified with their CD11b positivity when immunostained with antiCD11b-FITC conjugated antibody. Isolated groups from different groups show irregular shapes and variations. Representative figure of phase contrast of microglia isolated from young adult shows both amoeboid and ramified microglia with slender projections [Figure 4A]. These morphological variations are also supported by immunostaining. In the case of an adult, CD11b+ cells show both irregular and elongated morphology [Figure 4B], whereas isolated cells from neonates show rounded/irregular structures [Figure 4C]. Trypan bluestaining, separate from immuno-staining, was used to discriminate between viable (unstained) and non-viable cells (blue) not shown, was done to determine the yields of isolated, viable cells (in percentage) from different age groups [Table 1].

Evaluation of the ultrastructural changes of microglial cells isolated from brain tissue

Morphological variations of microglia become prominent when isolated microglia from different groups is studied ultrastructurally. Microglial cells isolated from neonates show rounded/irregular morphology, whereas those isolated from adult brain appear as irregular, elongated morphology with variations. These morphological variations among different age groups are also supported by further ultrastructural analysis. Cellular architectures of microglia isolated from brain tissue are determined by scanning electron microscopy (SEM). SEM studies reveal the population of microglial cells in developing early embryonic brain. Most of the isolated cells are irregularly round with serrated surface, typically monocyte/macrophage morphology in this stage of 


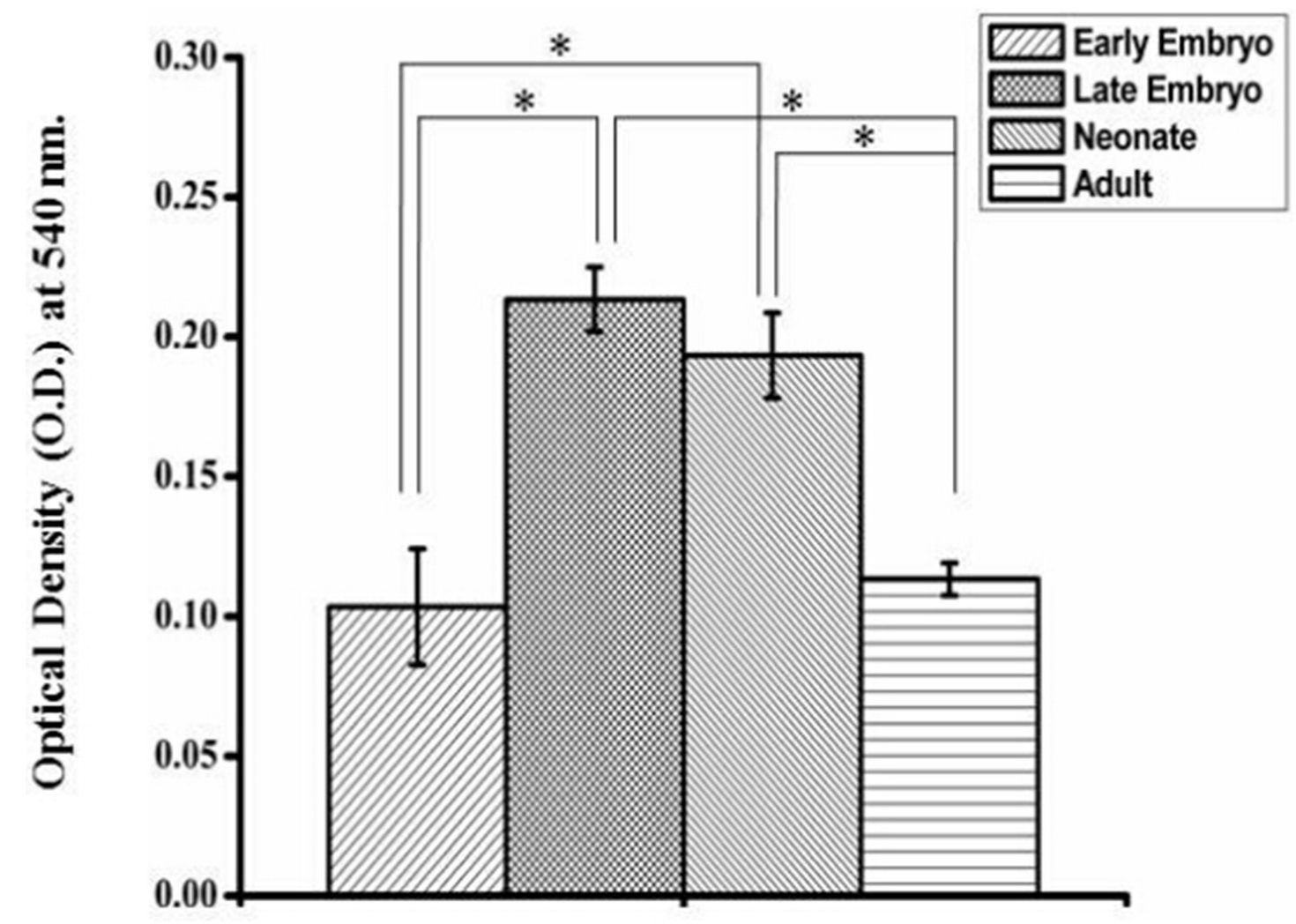

\section{Phagocytosis by NBT assay}

Figure 7: Quantification of phagocytosis using a colorimetric NBT assay. ROS generation by cells from different age groups in normal conditions was measured by using the colorimetric NBT assay done in triplicate set. Data are statistically interpreted by using one-way ANOVA followed by Tukey-Kramer test used for post-hoc multiple comparisons where $P<0.05$ is considered significant. Significant difference associated with phagocytic ability present between different age groups where $P$ value is less than 0.05 indicated by * symbol in graph. Graphical representation shows there is a significant increase of phagocytic ability in both late embryo and neonates as compared to the early embryo (indicated by * mark) whereas in case of adult the variation is insignificant with early embryo. Significant difference in phagocytic potential between these two groups (late embryo and neonates) with the adult is also indicated by * symbol

developing brain [Figure 5A]. This pattern [Figure $5 \mathrm{~B}]$ is gradually changing where the amoeboid cells appear with extended pseudopodia or cellular projections in the late embryonic phase. Figure 5C reveals the presence of both amoeboid microglia and the extended structure of a microglial cell. Both of them are showing active phagocytic forms. When an animal develops from infant to adulthood its microglial structure changes from rounded to elongated ramified forms. In an adult [Figure 5D], a cell shows an elongated structure with projection and a few slender filopodia that are prominent at the edge of the cell. This is the ramified, or so called resting, form that is characteristic of microglial populations observed in adult brains.

\section{Phagocytosis by microglia isolated from different age groups of animals}

Carbon particle ingestion shows the phagocytic activity in all stages from early embryo to adult. Carbon particle ingested or adhere to the cell is found in both embryos [Figure 6A and $\mathrm{B}$ ]. In neonates, phagocytic vacuole engulfing carbon particle in one cell and pseudopodial extension phagocytising carbon particle by another is visible [Figure 6C]. In case of adult, cell shows engulfed carbon particles along with a pseudopodial projection that entangle another carbon particle to draw and engulf [Figure 6D]. Measurement of phagocytosis in terms of oxidative burst is done in different age groups without any external stimulus and the animals maintained in normal condition. So the values of phagocytosis of different age groups reflect their baseline phagocytic capacity in normal condition. The OD value is directly proportional to their phagocytic capacity. This shows a prominent age dependent trend of phagocytic capacity. In early embryonic stage when they enter and populate in huge number to the brain parenchyma, they show less phagocytic potential. But it shows high phagocytic capacity at the late embryonic stage and continues for few days just after birth. Afterwards, during resting stage in stable adult brain this activity reaches to a basal level which is comparable to the early embryonic stage [Figure 7]. Statistical interpretation of the data by one way ANOVA, followed by Tukey-Kramer test used for post-hoc multiple comparisons, shows that there is a 
significant variation in phagocytic capacity between early embryo and both late embryo and neonates in phagocytic potential or oxidative burst in normal condition. But the difference between late embryo and neonates is insignificant. In contrast, there is a significant difference in phagocytosis between these two groups (late embryo and neonates) with the adult. Thus, it shows the trend of high phagocytic capacity just before and after birth, while early adulthood microglial cells become inactive in terms of phagocytosis, therefore, transformed into a resting or surveillance stage.

\section{DISCUSSION}

Our present study revealed a shift of microglial structure, distribution, and function across preand postnatal stages of development in the rat brain under normal physiology. This transverse analysis of microglial activities in brains from embryos to adults shows that microglia are capable of dynamically adjusting their position, shape, and function as the developmental requirements of the CNS change. If an age related structure-function model of microglia has been determined, deviations from it may be diagnostic for some CNS disorders. However, this study is limited to the developmental maturation of the rat brain, mostly up to young adulthood, which excludes the aging brain.

Our study shows significant differences in the morphology of microglial cells throughout the developmental/age phases, along with their functional attributes. Initially, by $\mathrm{HE}$ staining, round shaped macrophage/myeloid lineage cells, in both embryonic and infant stages, were found in brain parenchyma, whereas in adults, these cells showed conversion into elongated structures. Throughout the developing brain parenchyma, a particular colonizing pattern of bloodborne myeloid cells was observed as they migrated from the inner ventricular margin to the outer cortex region. In late embryonic and infant stages, few cells were observed to enter the brain from blood vascular fenestrations. However, in adults, normal HE staining revealed the presence of monocytes, many of which were present at the margin and tethered to the endothelium, in the perivascular space infiltrating from the capillary into deep brain parenchyma. Thus, while transitioning to adulthood, cells of the myeloid lineage stabilize their positions in the CNS and develop their morphological attributes. Furthermore, although the general notion of microglial populations being fixed in normal adult brains, ${ }^{[7,8,30,31]}$ the present micrographs indicate a potential avenue for blood-borne, myeloid-monocytic cells to enter the brain parenchyma.

Ultrastructural study by SEM of isolated cells also revealed the structural changes. Microglia recovered from brains of different developmental ages show distinctive trends in morphology. Many of them retain spine bearing surfaces that radiate from the cell body, a feature also seen in micrographs of isolated cells. A few cells remain amoeboid in shape with a ruffled surface membrane and short stubby projections. Ramified or “resting” microglia with extended pseudopodia, isolated from adult brains, were also found, which in turn gives us information about the amoeboid to ramified transition throughout the various developmental phases or ages. ${ }^{[32,33]}$ With the maturation from embryo to adult, the shift from amoeboid to ramified morphology, is supported prominently in the silver-gold and SEM studies. The microglia/macrophage specific CD11b marker expression, determined by immunofluorescence, in isolated cells confirms their lineage. Phagocytosis of ultrafine carbon particles by isolated monocytic/myeloidlineage cells occurred at all stages, from early embryo to adult, but the efficacy varied among the different groups. The concept of a microglial dichotomy with M1 and M2 phenotypes $^{[34]}$ may be examined with this model. The NBT assay, for estimating ROS generation, documented mild phagocytic activity in the early embryo and adult and much higher phagocytic activity in late embryos and neonates. This difference of phagocytic activity during the perinatal period is functionally related with the developmental organization of brain tissue. In that stage, neuronal positioning and extension, formation of neuronal circuitry, abrogation of wrong connections and reformation of proper contacts, and synaptic pruning are at their highest levels to develop a properly functional CNS system. ${ }^{[13,15,35,36]}$ Thus, there are enough phagocytic activities to trim and clean the forming CNS during the perinatal phase. ${ }^{[12,35,37]}$ Furthermore, phagocytosis at a controlled pace has an active and important role in developing and maintaining proper organization and integrity of CNS tissue for the lifetime of the animal. Hence, our study showed that the functionally linked increase in levels of basal phagocytic activity in microglial cells isolated from brains, before and after birth, were present mostly in cells showing an amoeboid morphology. The overall information gathered from our study is a morphological, functional relationship of brain macrophage/microglia in normally developing rat brains with a significant pattern of colonization in both early and late embryo, neonates, and even in adults. This study documents the spacio-temporal activities of versatile, immune-competent brain cell sunder normal physiological conditions from development to maturity. These baseline activities may be used as a reference frame for detecting and analysing morphological, functional anomalies of microglia in deformities and disease.

In summary, our results show an age-dependent variation of morphological and behavioural functioning 
of microglia, which act as sentinels of the CNS. Normal HE staining and specialised silver-gold staining of brain tissue show morphologic variations of these cells in both pre and postnatal stages of the developing rat brain, which are further supported with an ultrastructural, SEM study. The microglial identity of the isolated cells was confirmed with a CD11b marker. The cells in the normally developing brain also show an age dependent variation in their baseline phagocytic capacity, which is higher in the perinatal phase and assumed to be related with the normal physiology of CNS development and function. Therefore, changes in age sensitive microglial morphology and phagocytic activity from the embryonic to the adult brain could be an important reference when considering any neuropathological conditions with microglial involvement.

\section{Acknowledgments}

The authors are grateful to Dr. Srikanta Chakroborty of the USIC, Burdwan University, WB, India for providing technical support for SEM.

\section{Financial support and sponsorship}

The work is financially supported by UGC-MRP Grant [No. F. PSW-169/09-10 (ERO)] and by DST- YS (SERB) Project Grant [No.- SR/FT/LS-81/2011], Govt. of India.

\section{Conflicts of interest}

There are no conflicts of interest.

\section{REFERENCES}

1. Ginhoux F, Lim S, Hoeffel G, Low D, Huber T. Origin and differentiation of microglia. Front Cell Neurosci 2013;7:1-14.

2. Perry VH, Gordon S. Macrophages and microglia in the nervous system. Trends Neurosci 1988;11:273-7.

3. PerryVH, Gordon S. Macrophages and the nervous system. Int Rev Cytol 1991;125:203-44.

4. Ling EA, Penney D, Leblond CP. Use of carbon labelling to demonstrate the role of blood monocytes as precursors of the 'amoeboid cells' in the corpus callosum of postnatal rats. J Comp Neurol 1980;193:631-57.

5. Streit WJ. Microglia and macrophages in the developing CNS Neurotoxicol 2001:22:619-24.

6. Hanisch UK. Microglia as a source and target of cytokines. Glia 2002:40:140-55

7. Graeber MB, Streit WJ. Microglia: Immune Network in the CNS. Brain Pathol 1990;1:2-5.

8. Becher B, Prat A, Antel JP. Brain-immune connection: immuno-regulatory properties of CNS-resident cells. Glia 2000;29:293-304.

9. Nimmerjahn A, Kirchhoff F, Helmchen F. Resting microglial cells are highly dynamic surveillants of brain parenchyma in vivo. Science 2005;308:1314-8.

10. Kettenmann H, Hanisch UK, Noda M, Verkhratsky A. Physiology of Microglia. Physiol Rev 2011;91:461-553.

11. Luo XG, Chen SD. The changing phenotype of microglia from homeostasis to disease. Transl Neurodegener 2012;1:9.

12. Neumann H, Kotter MR, Franklin RJ. Debris clearance by microglia:an essential link between degeneration and regeneration. Brain 2009;132:288-95.
13. Paolicelli RC, Bolasco G, Pagani F, Maggi L, Scianni M, Panzanelli P, Giustetto M, Ferreira TA, Guiducci E, Dumas L, Ragozzino D, Gross CT. Synaptic pruning by microglia is necessary for normal brain development. Science 2011;333:1456-8.

14. Tremblay ME, Lowery RL, Majewska AK. Microglial interactions with synapsesaremodulated by visual experience.PLoSBiol2010;8:e1000527.

15. Tremblay ME, Majewska AK. A role for microglia in synaptic plasticity? Commun Integr Biol 2011;4:220-2.

16. Wake H, Moorhouse AJ, Jinno S, Kohsaka S, Nabekura J. Resting microglia directly monitor the functional state of synapses in vivo and determine the fate of ischemic terminals. J Neurosci 2009:29:3974-80.

17. Hanisch UK, Kettenmann H. Microglia: active sensor and versatile effector cells in the normal and pathologic brain. Nat Neurosci 2007;10:1387-94

18. Kim SU, de Vellis J. Microglia in health and disease. J Neurosci Res 2005;81:302-13

19. Block ML, Zecca L, Hong JS. Microglia-mediated neurotoxicity: uncovering the molecular mechanisms. Nat Rev Neurosci 2007;8:57-69.

20. Kreutzberg GW. Microglia: a sensor for pathological events in the CNS. Trends Neurosci 1996;19:312-8.

21. Ghosh A, Mukherjee J, Bhattacharjee M, Sarkar P, Acharya S, Chaudhuri $\mathrm{S}$ and Chaudhuri S. The Other Side of the Coin: Beneficiary Effect of 'Oxidative Burst' Upsurge with T11TS Facilitates the Elimination of Glioma Cells. Cell Mol Biol 2007;53:53-62.

22. Ochiogu IS, Uchendu CN, Ihedioha JI. A new and simple method of confirmatory detection of mating in Albino rats (Rattus norvegicus). Animal Research International 2006;3:527-30.

23. Penfield W, Cone W. Neuroglia and microglia (the metallic methods). In: McClung CE, editer. In Handbook of Microscopical Technique. New York: Paul B Hoeber Inc.; 1937.p. 489-521.

24. McCarter JC. A Silver Carbonate Staining method for oligodendrocytes and microglia for routine use. Am J Pathol 1940;16:233-235.2.

25. Begum Z, Sarkar S, Mukherjee J, Ghosh A, Chaudhuri S, Chaudhuri S. Evaluation of anti-tumor property of specific and nonspecific BRMS in experimental glioma by assessing the microglial cell functional and phenotypic modulations. Cancer Biol Ther 2003;2:356-63.

26. Ford AL, Goodsall AL, Hickey WF, Sedgwick JD. Normal adult ramified microglia separated from other central nervous system macrophages by flow cytometric sorting. Phenotypic differences defined and direct ex vivo antigen presentation to myelin basic protein-reactive CD4+ T cells compared. J Immunol 1995;154:4309-21.

27. Chen X, Zhang Y, Sadadcharam G, Cui W, Wang JH. Isolation, purification, and culture of primary murine microglia cells. Bio-protocol 2013;3:1-6.

28. López-Cepero JM. Silver Carbonate staining reveals mitochondrial heterogeneity. J Histochem Cytochem 2004;52:211-6.

29. Esiri M, Perl D. Oppenheimmer's diagnostic neuropathology: a practical manual. 3rd ed. London:Hodder-Arnold; 2006.

30. Mildner A, Schmidt H, Nitsche M, Merkler D, Hanisch UK, Mack M, Heikenwalder M, Bruck W, Priller J, Prinz M. Microglia in the adult brain arise from Ly-6ChiCCR2+ monocytes only under defined host conditions. Nat Neurosci 2007;10:1544-53.

31. Carson MJ, Doose JM, Melchior B, Schmid CD, Ploix CC. CNS immune privilege: hiding in plain sight. Immunol Rev 2006;213:48-65.

32. Giulian D, Baker TJ. Characterization of amoeboid microglia isolated from developing mammalian brain. JNeurocsi 1986;6:2163-78.

33. Streit WJ, Walter SA, Pennell NA. Reactive microgliosis. Prog Neurobiol 1999;57:563-81.

34. Mittelbronn M. The M1/M2 immune polarization concept in microglia: a fair transfer? Neuroimmunol Neuroinflammation 2014;1:6-7.

35. Schafer DP, Stevens B. Synapse elimination during development and disease: immune molecules take centre stage. Biochem Soc Trans 2010;38:476-81.

36. Schafer DP, Lehrman EK, Stevens B. The "Quad-Partite" synapse: Microglia-synapse interactions in the developing and mature CNS. Glia 2013;61:24-36.

37. Harry GJ, Kraft AD. Microglia in the developing brain: A potential target with lifetime effects. Neurotoxicology 2012;33:191-206. 